\title{
Oral anticoagulant treatment in patients with mechanical heart valves: how to reduce the risk of thromboembolic and bleeding complications
}

\author{
S. C. CANNEGIETER', M. TORN' \& F. R. ROSENDAAL' '

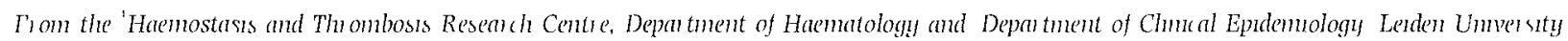 \\ Medical Centre. the Netherlands
}

\begin{abstract}
Cannegieter SC, Torn M, Rosendaal FR (Leiden University Medical Centre, the Netherlands). Oral anticoagulant treatment in patients with mechanical heart valves: how to reduce the risk of thromboembolic and bleeding complications (Minisymposium: Oral anticoagulants). J Intern Med 1999; 245: 369-374.

Patients with mechanical heart valves have a high risk of thrombus formation on the valve and subsequent systemic embolism. These patients therefore need to receive life-long oral anticoagulation $(O A C)$. Despite this treatment, the overall incidence rate of major thromboembolic complications is still about 1-2 per 100 patient-years. Additionally, these paticnts have an increased risk of bleeding complications, ranging between 1 and 7 per 100 patientyears.

To reduce both types of often very serious complications, the optimal intensity of anticoagulation needs to be established. We found a fairly wide optimal range between 2.5 and 4.9 INR (international
\end{abstract}

\section{Life-long anticoagulation}

Mechanical heart valve prostheses have a high thromboembolic potential. This can be partly attributed to the inorganic material they are made of, but is also a result of nonphysiological blood flow normalized ratio) at which the incidence of both untoward events was minimal. As a target intensity, we recommend opting for the middle of this range (INR 3.0-4.0), thereby providing a safe margin at both ends.

In order to further reduce thromboembolic and bleeding complications, two approaches can be considered: first of all, the management of $\mathrm{OAC}$ treatment needs to be optimized in order to achieve a stable therapeutic effect in as many patients as possible. Secondly, patient characteristics need to be identified that increase the thromboembolic or bleeding risk. Subsequently, the optimal intensity may need to be adjusted accordingly, at an individual level. Possible risk factors for an increased thromboembolic risk are position and type of the prosthesis. Age may increase both the risk of thromboembolism and the risk of haemorrhage.

Keywords: haemorrhage, heart valves, oral anticoagulation. patterns around the prosthesis, with areas of turbulent flow and relative stagnation. The risk of thrombus formation on the valve and subsequent embolism without any antithrombotic treatment averages about $10 \%$ per year [1]. This is in contrast to patients with biological prostheses whose throm- 
boembolic risk is not considered to be increased (after the first 3 months) in the absence of other risk factors such as atrial fibrillation or an enlarged left atrium [2]. There is therefore no doubt that patients with mechanical heart valve prostheses need to receive life-long oral anticoagulation (OAC), which, unfortunately, is associated with an increased risk of hemorrhagic complications.

\section{Optimal intensity}

Despite the use of OAC, the overall incidence rate of major thromboembolic complications is still about 1-2 per 100 patient-years [1]. Major bleeding events range between 1 and 7 per 100 patientyears [2]. In a follow-up study of 1608 unselected patients with a mechanical heart valve treated in regional anticoagulant clinics, we found that the risk of any major adverse event (all thromboembolism or bleeding combined) was 3.5 per 100 patientyears [3]. The most feared complication is stroke, whether it is thromboembolic or haemorrhagic. In our study, we found an incidence of intracranial bleeding of 0.5 per 100 patient-years and of cerebral infarction of 0.7 per 100 patient-years. Seventy-two per cent of these patients died or suffered lasting neurological impairment.

For many indications for oral anticoagulant therapy it has been established that with more intense anticoagulation, thromboembolism is progressively prevented, whilst the risk of bleeding increases [3-6]. Consequently, a level of anticoagulation can be found at which both complications are minimal, i.e. the optimal intensity of OAC. For mechanical heart valves, we found this optimum between 2.5 and 4.9 INR [3]. Within this range, the incidence of all untoward events was about two per 100 patient-years, rising to extreme values below and above: from an incidence of 7.5 per 100 patient-years for INR 2.0-2.5, up to an incidence of 27 per 100 patient-years at the very low range of 1.0-1.4. At the other end, the incidence of all events rose steeply again, from 4.8 per 100 patient-years at INR 5.0-5.5 up to 75 per 100 patient-years at INRlevels over 6.5 (Fig. 1). Since the risk of any untoward event hardly varied between an INR of 2.5 and 4.9 , we recommend choosing a target intensity lying in the middle of this range (INR $3.0-4.0$ ), thereby providing a safe margin at both ends.

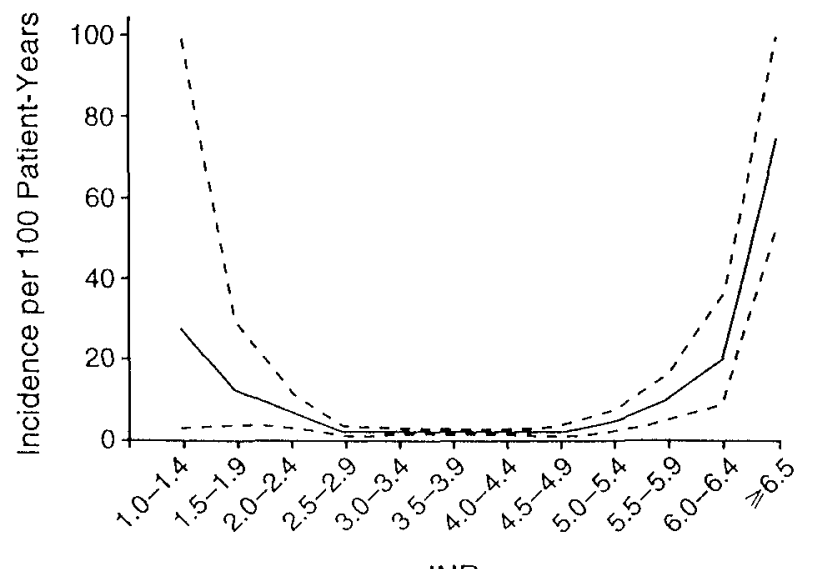

INR

Fig. 1 INR-specific incidence of all adverse events (all episodes of thromboembolism, all major bleeding episodes and unclassified stroke). The dotted lines indicate the $95 \%$ confidence interval. (Reproduced with permission from $N$ lingl / Med 1995: 333: 11-17.)

\section{Optimal treatment: further reducing com- plications}

If we want to aim our efforts at further reducing the number of both thromboembolic and bleeding complications, two approaches can be considered. First of all, we can concentrate on the patients who have INR values below and above the optimal range (and are therefore at high risk of either thromboembolism or bleeding), and question why their treatment apparently is inadequate. Secondly, we must realize that, although the risk is lowest within the optimal range, the absolute number of events that occurs here is still considerable (since the majority of the patients are within this range most of the time). Therefore, we should try to assess what other factors contribute to the occurrence of stroke, despite the fact that $\mathrm{OAC}$ treatment is adequate.

\section{Improving anticoagulant treatment}

Oral anticoagulant treatment is achieved with coumarin derivatives, vitamin K-antagonist drugs which impair thrombus formation to an extent that depends on the coumarin dose. However, large discrepancies exist in dose and anticoagulant response between subjects. Besides, these variations also occur in the same patient during long-term treatment. As a consequence, the intensity of the anticoagulant treatment needs to be measured 
regularly and, if necessary, the dosage adjusted. The way this is organized differs largely between countries; in the Netherlands specialized anticoagulation clinics have been emerging since the 1950s. and countries such as Italy, Canada and the United States have since followed. Monitoring by these clinics clearly improves the quality of care [7]. Still, even within such a setting, only about $6(--70 \%$ of the intensity measurements are found to be within the target range, about $20-30 \%$ are below and $10 \%$ above the upper range [3, 8]. Many factors may contribute to this variability, such as changes in diet (availability of vitamin K) [9], poor patient compliance [10]. comedication [11] and intercurrent diseases. Possible strategies to improve the amount of time spent within the target zone were recently described. Weibert et al. showed that administering $2.5 \mathrm{mg}$ of oral vitamin $\mathrm{K}$ rapidly corrects excessive anticoagulation (INR $>5.0$ ) without inducing resistance to further anticoagulation [12]. Ansell et al. proposed introducing patient self-management of their OAC dose, and showed in their study that this can be a successful approach [13]. Van der Meer et al. showed in a pilot intervention study that it is possible to improve compliance by special attention and devices $[10]$.

\section{Subgroups with different risk profiles}

\section{Risk factors for thromboembolism}

Associated with the prosthesis. All currently available types can be classified into three broad categories with largely different flow characteristics [14]: the caged ball types (Starr-Edwards); singletilting disk valves (Björk-Shiley, Sorin, Medtronic Hall, Omnicarbon); bileaflet valves (St Jude, DuroMedics, CarboMedics). Only one study has compared directly in a randomized design possible differences in thromboembolic risk between these valve types [15]. In this study a higher thromboembolic risk was found in patients with a bileaflet model (EdwardsDuromedics) and in patients with a Medtronic Hall valve, compared to Björk-Shiley valves. However, it has been debated whether these discrepancies should be attributed to different thrombogenicity [16]. In a meta-analysis of 46 observational studies on thromboembolic complications in patients with mechanical heart valves, we found a higher risk of embolic complications in patients with a caged-ball valve [1]. Compared to patients with a caged ball valve, the incidence of total thromboembolism was $30 \%$ lower for patients with a tilting disk valve (RR $0.7(95 \%$ CI $0.5-0.9))$, and $50 \%$ lower for patients with a bileaflet valve (RR $0.5(95 \%$ CI $0.4-0.7)$ ). In the cohort study of 1608 patients with mechanical heart valves, we also found a clearly higher risk of thromboembolism in patients with caged-ball valves. which was independent of other factors such as age or position of the valve. An incidence of 0.5 per 100 patient-years was found for the bileaflet valves. of 0.7 per 100 patient-years for the tilting disk valves, and of 2.5 per 100 patient-years for the caged-ball valves [3]. The position of the prosthesis (mitral, aortic or both) also influences the thromboembolic risk. The results of the meta-analysis showed that the risk of major embolism was about doubled in patients with a mitral prosthesis |1]. In the cohort-study, we found similar differences. In patients with an aortic prosthesis the incidence of thromboembolism was 0.5 per 100 patient-years, in patients with a mitral valve it was 0.9 per 100 patient-years, and 1.2 per 100 patient-years in those with both an aortic and a mitral valve [3].

Associated with other cardiac disease. When effective mechanical atrial activity is impaired, blood stasis may enhance the development of atrial thrombi and thus increase the risk of systemic embolism. This may occur in atrial fibrillation and left atrial enlargement. It is difficult to assess to what extent the increased risk of a mitral prosthesis can be explained by atrial abnormalities or vice versa, since they are strongly associated. Burchfiel et al. found a threefold higher incidence of systemic thromboembolism in patients with an enlarged left atrial dimension [17]. However, this effect was entirely explained by the location of the prosthesis (mitral versus aortic), when this was taken into account in a multivariate analysis [17]. Contradictory results have been published on atrial fibrillation. Burchfiel et al. found an independent increased risk in these patients, whilst Butchart et al. did not [18].

Other risk factors. It is not clear to what extent established risk factors for ischaemic stroke in the general population have an additional effect on the high risk already present in artificial heart valve patients. This is probably caused by a lack of sufficient numbers of stroke patients within study 
groups to allow for statistically solid conclusions on subgroups. We found an effect of age on the risk of thromboembolism [3]. Of all 45 events, only one occurred in a patient younger than $50(0.1$ per 100 patient-years), and this risk rose to 0.8 per 100 patient-years in patients between 50 and 70 and up to 1.1 per 100 patient-years in patients older than 70 . Butchart et al. found hypertension to be a strong risk factor in patients with aortic valve replacements, as well as smoking [18]. Cortelazzo et al. reported an increased risk of thromboembolism in patients who had a history of such a complication [7].

\section{Risk factors for bleeding}

There have been many reports on risk factors for bleeding in anticoagulated patients, although most have done so only for total groups of patients anticoagulated for a variety of indications. Unfortunately, these studies create some confusion about risk factors for bleeding. First of all, conflicting results have been reported about the effect of age on bleeding risk. About an equal number of papers have described how elderly patients are at higher risk for haemorrhagic complications [3, 19-23], as have failed to find such an association [7, 24-27]. Contradictory findings have also been reported on the effect of hypertension. Most authors did not find a clear association $[7,26,28,29]$. Only Launbjerg et al. described a slightly increased bleeding risk in patients with hypertension [21]. Cortelazzo et al. found increased bleeding risk in patients who had had a previous haemorrhage [7]. Landefeld et al. also found that a history of gastrointestinal bleeding increased the risk of major bleeding about threefold [20]. This effect has not been described by other authors. They also described a similar influence of the presence of a serious comorbid condition such as recent myocardial infarction, renal insufficiency or severe anaemia. Fihn et al. reported only a slightly increased effect of the presence of three or more comorbid conditions [29]. Gitter et al. found the presence of malignancy to be associated with bleeding risk [26].

On the whole, these results are quite contradictory. An explanation for the discrepancies is difficult to provide. Several mechanisms may be plausible as described by Beyth \& Landefeld [30], for example a lack of statistical power to detect a difference due to a limited number of events in the subgroups. Furthermore, it could be due to selection bias, since in a trial setting as well as in an observational study, patients with perceived high bleeding may be withheld from treatment with OAC.

\section{Optimal intensity for subgroups}

When it is known that a certain risk factor increases the thromboembolic or the bleeding risk, it needs to be assessed how this affects the optimal anticoagulation level. Two scenarios may be considered, either the incidence rate of the adverse event is increased to the same extent at every INR level (in which case the optimal level does not change), or the risk increases only at lower intensities for subgroups with increased risk of thromboembolism, and only at higher intensities for subgroups with increased risk of bleeding. In our cohort study we investigated if and how the optimal level changed according to age, valve position and valve type [3]. The results showed that when an increased thromboembolic risk had been established for a subgroup (e.g. mitral versus aortic valve), this was associated with a higher incidence of thromboembolism only at the lower INR levels ( $2-3$ INR), in other words, the optimal level was narrowed on the left side of the curve. The same applied for the only subgroup in which an increased bleeding risk was found (age older than 70), in whom the incidence of bleeding was increased only at higher INRs (4-5 INR), compared to the younger age groups. In short, the difference between subgroups manifested itself only at the extremes of the optimal level. If this finding is confirmed in other studies, it may imply that the optimal level should indeed be adapted according to an individual's characteristics.

\section{Adding aspirin}

Some recent research has been aimed at further reducing thromboembolic complications by adding aspirin to the anticoagulant treatment $[31,32]$. Turpie et al. added low-dose aspirin (100 mg) to warfarin treatment (INR 3.0-4.5) and found a reduction of the embolic rates in the aspirin group, which was associated with an increased risk of major bleeding of 8.5 per 100 patient-years [31]. Meschengieser et al. compared $100 \mathrm{mg}$ aspirin added to less intense warfarin treatment (INR 
2.5-3.5), to warfarin alone (INR 3.5-4.5) and found a similar thromboembolic risk in both arms, whilst the bleeding risk was higher in the warfarin alone group [32]. These two trials offer interesting information but can not yet be considered as sufficient basis for recommending combination treatment (see below about the information gained from aetiologic and pragmatic studies).

\section{Further research}

More trials have recently been published comparing different intensity schemes $[33,34]$. The AREVA trial compared moderate anticoagulation (INR $2.0-3.0)$ to the usual regimen of INR 3.0-4.5. In this study a similar risk in thromboembolic events was found in both arms, whereas the bleeding risk was higher in the high intensity group [33]. Pengo et al. compared treatment with a target INR of 3.0 to a target INR of 4.0 and found similar results [34].

The question is what these trials (both the aspirin and the different regimes studies) contribute to our ultimate aim, i.e. reducing complications in these patients. Two types of studies can be distinguished in this context: (i) aetiological studies, in which research is aimed at detecting factors that determine the occurrence of an event; (ii) pragmatic studies, in which we want to assess the optimal practical strategy to actually decrease the risks. At an aetiological level, we feel that the effect of intensity of OAC treatment has been sufficiently studied [3]. In our study we calculated incidence rates of adverse events by taking the ratio of the number of events that actually occurred at a certain INR and the total number of patient-years that this INR had been achieved by the total patient population. They were therefore independent of the target level and purely represented the risk of an event when a certain INR is achieved. However, the patients in this study consisted of a large, heterogeneous group, so these results can only be applied to this group as a whole. Therefore, more research is needed to identify patient characteristics that increase or decrease either the thromboembolic or the bleeding risk and how they interact with OAC treatment. At a pragmatic level, for any other type of medication the trial approach would be the most informative in finding out the best regimen. However, since the management of anticoagulant treatment is so complicated and dependent on so many factors, it is very difficult to apply results from one centre to the other. In the Turpie trial, for example, the intensity of the anticoagulant control was within the target range only $40 \%$ of the time [31]. Even though they found a reduction of the risk of embolism when aspirin was added, it seems very unlikely that adding aspirin to anticoagulant treatment in the Netherlands would have the same effect, since the risk of complications there is already lower, thanks to well controlled OAC treatment. Another problem with trials in this field is the oversimplified comparison between bleeding and thromboembolic events. A thromboembolic complication is usually much more severe than a bleeding, especially in terms of permanent disability. The AREVA trial, for example, was stopped when the bleeding risk was significantly higher in the high than in the low intensity arm, whereas the thromboembolism risk was equal in both [33]. However, this was only due to an increase in minor haemorrhagic events, whilst the risk of major bleeding was not different. Minor events were defined as 'all non-major bleeding', which hardly seems comparable to thromboembolic events. Since bleeding (especially defined like this) is much more common than thromboembolism, this inevitably results in a situation where any possible difference in thromboembolic rate can not be detected. Pragmatically, therefore, instead of performing an endless series of trials comparing different intensities, it is probably more efficient to establish ways of improving $\mathrm{OAC}$ treatment at a local level and to find ways to keep as many patients as possible within the optimal range.

\section{Conclusion}

Unfortunately, patients with prosthetic heart valves need to be treated with $\mathrm{OAC}$ for the rest of their lives. Cumulatively, they have a considerable risk of 'ever' experiencing a serious thromboembolic or bleeding complication. We need to continue searching for ways to prevent these. Further research should be aimed at finding ways to optimize the management of OAC treatment and at identifying patient characteristics that increase the thromboembolic or bleeding risk.

\section{References}

1 Cannegieter SC. Rosendalal FR. Brièt E. Thromboembolic and 
bleeding complications in paticnts with mechanical hout ialve prostheses Cuculation $1994 \quad 89635-41$

2 Stem PI) Alpeit Is Copcland J Dalen JF Goldman S I uipic AGG Antithombotic theiapy in paticnts with mechanical. and biological prosthetic heal valves chost $1992 \quad 102$ 445-55s

3 Cannegieter SC Rosendad FR Wint/cn AR van dei Meel ГJM Vandenbroucke JP Biıet L Optimal ord anticoagulant theiapy in patients with mechanical heait valies $N$ I nal I Med $1995 \quad 333 \quad 11-17$

4 The I uropedn Atıd ribillation Tilal Study Gioup Optimal oral anticodgulant theipy in patients with nomheumatic

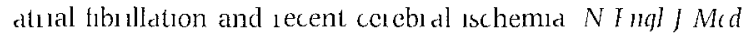
1995333510

5 A ar AJ Cannegicter SC Deckes JW at al Optimal intcnsity of oral anticodgulant thesapy alter myocadial inlaction I Am coll caldol $199627 \quad 1349-55$

6 The stıoke Pievention In Reversible Ischemid Tridl (SPIRIT) study Goup A tandomized trial of anticodgulants versus aspitin after cesebial ischemia of prcsumed artestal osigin Amil Vallol $1997 \quad 42 \quad 857-65$

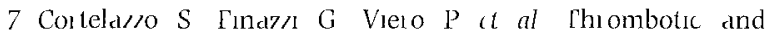
hemorhagic complications in patients with mcchanical heat valie prosthesis attending an anticoagulation clinic I/nomb Hacmost $199369 \quad 316-20$

8 Rosendal IR van de Meer IJM Cannegreter SC Manage ment of anticoagulant therapy the Dutch experience I Thromb Thrombolyss $19962265-9$

9 Paterson PF Kwaan IC Curient concepts of wollatin therapy Alch Intcun Mcd $1986 \quad 146 \quad 581-4$

10) Van der Meer ГjM Briet E Vandenbroucke JP Slamck D] Versluys MHPM Rosendaal HR The role of comphance as a Cause of instability in ol al anticoagulant therapy Bi J Hacmat $1997 \quad 98 \quad 893-900$

11 Haide S Thuimann P' Clinically impoitant drug mtciactions with anticoagulants an update Clincal Phomacolmotics $199630 \quad 416-44$

12 Weibeit RI I e DT Kaysel SR Rapaport SI Correction of excessive anticoagulation with low-dose olal vitamin $\mathrm{K}$ Am Inton Mcd $1997 \quad 125 \quad 959-62$

13 Ansell JL Patel $N$ Ostrovsky D Norzolillo L Peteison AM Fish L Long tcrm patient self management of oral antic Odgulation Alch Intch Mcd $1995155 \quad 2185-9$

14 Vongpatanasm W Hillis D Lange RA Prosthetic heart valves N I nal J Mcd $1997335407-16$

15 Kuntse $\mathrm{CF}$ Ebcls 1 Ejjgeldar A Homan Van do Hode JN Rates of thromboembolism with thiee dilferent mechanical heart valve prostheses I andomised study I anct 19898637 51417

16 Lewis PA Butchart $\mathrm{EG}$ I ong-term evaluation of mechanical heart valves Ianat 198986451020 (letter)

17 Burchfiel CM Hammermeister KF Krause Steinrauf H a $a l$ Left atrial dimension and risk of systemic embolism in paticnis with a prosthetw heart valve I Am coll caldol 1990 15 32-41

18 Butchart $\Gamma G$ de Moreno la Santa $P$ Rooncy SJ Lewis PA Arterial risk tactors and ischcmic cerebrovasculal cvents after aortic Ialve ieplacement / Hoart Valve Dis $1995 \quad 4 \quad 1-8$

19 Van de Meer ГJM Rosendad FR Vandenbroucke JP Briet L Assessment of a bleeding risk index in two cohorts of patients treated with oral anticoagulants Thromb Hacnost 199676
1216

20 I andetald (s Goldman I Maljor bleeding in outpalicuts treated with ualain madence and piediction by factors known at the stat of outpatient the apy $A m / M a d 198987$ 144-52

2] I cumbjerg J Lgeblad H Hcal' I Nielsen NH I ugleholm $\triangle M$ I adctoged K Bleeding complications to oral anticoagulant thadpy multivalatc analysls of 1010 teatment ycas in 551 oulpatients / Inton Mod 1991 $229351-5$

22 Walarin icrsus aspuin for prcvention of thromboembolism in atial fibullation Stioke Picvention un Airal I ibillation Il sudy Iamet 199434368791

23 Palarelı G I calı N Coccheis stal Blceding complications of oral an1icoagulant tieatment an mecption coholt prospec tuve collabol atuve study (ISCOA I) I ancet $1996 \quad 348 \quad 423-8$

24 Pcily GW I cmnhan L Mohi JP a al complications of long term anticodgulation Am Vamol $198823 \quad 57074$

25 curwil/ Jlt Goldbag RJ Holden A Knapic N Ansell J Agc relatcd risks of long tcim or al rnticodgulant thel apy Aich Intron Med $1988 \quad 148 \quad$ ]733-6

26 Gittel MJ Jacger IM Pcticison IM Gersh BJ Sllvcistem Ml) Bleeding and thomboembolism duing anticorgulant thet apy a population based study in Rochester Minnesola Malo Clin Ploc $199570725-33$

27 I shn SD Cxlleshan (M Martin DC McDonell MB Fenılsoll Ju, White RH the risk and scicity of blecding complications in cldesly patuents tieatcd with ualatm Ann Intom Mod 1996 12497079

28 Hylek LM Singel DI Risk factors lon mil aciantal hemon hage in outpatients taking $u$ alim Am Jntem Med 1994120 897902

29 I Ihn SD McDonell M Matin D a al Risk factors for complications of chionic anticodgulation Ann Intin Med 199311851120

30 Bcyth RJ I andeleld (s Outcomes of u aldal in thel apy Iessons flom the ical world Mayo (Im Ploc 199570 806-8(editol lal)

31 I us ple $\Lambda G$, Gent $M$ Laupacis $A$ at al $\Lambda$ compalison of aspirin with placebo in patients lieated with watalin aflu hedit valve ieplacemcont $N$ l ngl J Mcd $1993329524-9$

32 Meschengieser \&s Fondevilla (G, I lontroth ] Santal ell Ml I a/7ar MA Low intensity ol al anticoagulation plus low dose aspirin versus high intensity or al anticodgulation alone a iandomized trial in patients with mechanical prosthetic heat valves I Fhorac Cardovasc Sua $1997113910-16$

33 Acar J Iung B Borssel JP a al ARFVA Multicentel iandomied trial comparison ol low dose versus standaid dosc anticoagulation in patients with mechanical piosthetic heart valves Cuculation 199694210712

34 Pengo $V$ Barbeio I Ban/ato $A$ a al $A$ compalison of a moderate with moderate high intensily oral anticoagulant Ircatment in patients with mochanical hedit valve prosthescs Ihomb Hacmost $1997 \quad 77 \quad 839-844$

Recoved 28 August 1998 accepted 7 Scptember 1999

Concspondene $\Gamma \mathrm{R}$ Rosendad Depatment of Clinical l pide miology Building 1 (O) P Iniversity Ilospital I eiden P() Box 9600 NI 2300 RC I cidcn the Netheilands (Iax +31715248122 e mall iosendad $\left(w_{1} u 1112\right.$ kidenuniv nl) 